OPEN ACCESS

\title{
A novel Electrospinning Procedure for the Production of Straight Âligned and Winded Fibers
}

Cesare Errico, Federica Chiellini, Nicola Detta, Anna Maria Piras, Dario Puppi, Emo Chiellini*

Laboratory of Bioactive Polymeric Materials for Biomedical \& Environmental Applications, UdR-INSTM, Department of Chemistry and Industrial Chemistry University of Pisa Via Risorgimento 35, 56126 Pisa, (Italy) Fax: +39 050 2210332, Phone number: +39 0502210301

*Corresponding author: emochie@dcci.unipi.it (Emo Chiellini)

\section{Abstract}

An electrospinning procedure allowing the spinning of a straight jet of polymer solution was developed. By using proper collector devices, it enables to collect winded and aligned fibers and to prepare polymeric constructs developing along the $Z$ axis. The reported results are expected to provide basic understandings on which parameters are controlling the stability/instability of the process and implement new applications of electrospinning with specific reference to the preparation of well defined three-dimensional structures.

Keywords:electrospinning, aligned fibers, polymers, punctiform deposition, controlled deposition

Citation: Cesare Errico et al. A novel Electrospinning Procedure for the Production of Straight Aligned and Winded Fibers. Nano Biomed.Eng. 2011,3(4), 222-226. DOI:10.5101/nbe.v3i4.p222-226

\section{Introduction}

Electrospinning is a method for producing fibers with diameters ranging from $10 \mu \mathrm{m}$ to $10 \mathrm{~nm}$ by accelerating a jet of charged polymer solution in an electric field [1]. Fibers form as a result of solvent evaporation and deposit on a collector as nonwoven fibrous mats that limits the applicability of the final constructs [2]. The controlled production of winded and straightly aligned fibers on a substrate and the preparation of three-dimensional devices are the major challenges in this area [3, 4]. Underlying the difficulties to achieve such control is the process of nanofibres formation itself, which mechanism is still matter of controversy.

In a typical electrospinning set-up the polymer jet follows a path that begins with a straight segment. The diameter of the jet straight segment decreases monotonically with the distance from the tip. Bending instabilities originate and grow rapidly into a coil (whipping motion), under the influence of the charge carried with the jet. Due to the growth of the whipping instabilities, the jet stretches and becomes thinner allowing the formation of the nanofibres [5].

Near Field Electrospinning (NFES) [6] and High Precision Deposition Electrospinning (HPDE) [7] are two techniques that made a breakthrough in this field, being able to control fairly precisely the location and deposition of the prepared nanofibres. These approaches involved the shortening of the distance the polymer travels in the electric field from the conventional 10-30 centimeters to few millimeters or less. This allowed to take advantage of the brief period of stability that polymer jet exhibits when the electrospinning process begins. However, the small volume of solution that can be spun at one time [8], the inability to prepare three-dimensional structures and to adequately remove solvent prior to collection [2] reduce the practicality of these processes.

In the present work, an electrospinning apparatus was developed that eliminates all process variables determining the onset of instabilities. This allows to spin a stable straight polymer jet in spite of the fact that the distance from the spinneret to the collector was kept up to several centimeters.

\section{Experimental}

\subsection{Materials}

Poly(lactic-co-glycolic acid) PLGA (75:25; mw $120 \mathrm{KDa})$ was purchased from Lakeshore Biomaterials Inc. (Birmingham, AL) and a $20 \mathrm{wt} \%$ solution in acetone or $30 \%$ in acetone/dimethylformamide (DMF) (3:2) were used. The electrospinning apparatus consisted of a $10 \mathrm{ml}$ syringe equipped with a 21 gauge blunt needle, a syringe 
pump (BSP-99M, Braintree Scientific Inc., Braintree, MA), two high voltage power supplies of opposite polarity (Spellman High Voltage, United Kingdom) and an RT collector (Linari Engineering).

\subsection{Electrospinning}

To obtain punctiform fiber deposition, power supplies were connected either to two parallel plates or two concentric cylinders, thereby forming the geometry of a capacitor, to ensure field homogeneity. The fluid was fed at a given flow rate to the metallic needle jutting out of a hole in the middle of the auxiliary plate of the outer cylinder. Both the polymer solution and the metallic needle were electrically insulated by a glass capillary from the power supplies. Polymer solution was electrospun according to the parameters following reported.

Parallel plates configuration: the distance between the plates was $9 \mathrm{~cm}$, the distance from the tip of the glass capillary to the collector was $4 \mathrm{~cm}$. Flow rate was $0.8 \mathrm{ml} \mathrm{h}^{-1}$ and the applied voltage $33 \mathrm{kV}$.

Concentric cylinders configuration: the cylinders diameter offset was $8 \mathrm{~cm}$, the diameter of the mandrel collector and the distance from the glass capillary tip to the collector were $3.7 \mathrm{~cm}$. Flow rate was $0.6 \mathrm{ml} \mathrm{h}^{-1}$ and the applied voltage $12 \mathrm{kV}$.

Fibers were collected onto the collector plate or onto an inner rotating mandrel. To enhance the evaporation of solvents an infrared lamp $(250 \mathrm{~W})$ was positioned above the jet paths during process as heat source.

\subsection{Electrospun Fibers Morphology}

The morphology of the electrospun fibre was examined by SEM (Jeol LSM 5600LV, Japan). The roto-translating mandrel was covered by an aluminium sheet that was removed after fibres deposition and sputter coated with gold particles. SEM pictures were acquired using the signal from the secondary electron detector.

\section{Results \& Discussion}

\subsection{Controlled deposition by using the parallel plates configuration}

For developing the present apparatus we analyzed the straightforward parallel electrode configuration first described by Shin et al to provide a theoretical description of the electrospinning process [9], and used by Bunyan et al to obtain a certain control over fibers deposition [10]. Parallel electrodes, consisting of a collector plate and an auxiliary plate, form the geometry of a capacitor with parallel electric field lines (Fig. 1a). Though the auxiliary plate was added to ensure operations in a uniform electric field, two main configuration features hampered the possibility to carry out the process in real uniform electric field. Both the presence of the charged metallic needle and the charged polymer jet between the plates interfered with the uniformity of the resulting electric field. In Fig. 1b is represented a schematization of the electric field generated by the described electrospinning set-up.

The modulus of the electric field $(E)$ is given by the ratio $V / d$ ( $V$ : potential; $d$ : distance between electrodes). Since the auxiliary plate and the needle were electrically connected, they were at the same potential. The distance between the needle tip and the collector plate $\left(d_{a-c}\right)$ was smaller than the distance between the plates $\left(d_{b-c}\right)$, thus the electric field generated between the needle and the collector plate $\left(E_{a-c}\right)$ was stronger than that generated

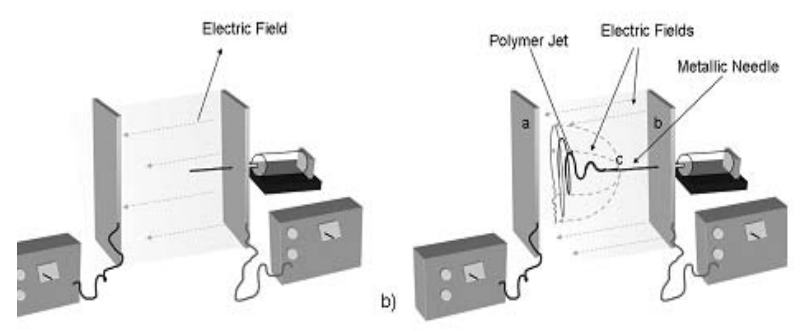

Fig.1 a) Schematization of a parallel electrodes electrospinning configuration. The electric field generated by the metal needle is neglected; b) Schematization of the electric field generated during the actual process of electrospinning by using the parallel plates configuration.

between the plates $\left(E_{a-b}\right)$. The resulting electric field was non-uniform and more similar to that of a typical needle-plate configuration rather than a parallel plates configuration. Furthermore, the charged polymeric while moving towards the counter electrode increased the unhomogeneity of the effective electric field.

The electrospinning apparatus we developed [11], solved in a simple way these two main interferences and allowed to eliminate the onset of bending instabilities commonly associated with any electrospinning process. A glass capillary was used to electrically insulate the metallic needle, and therefore the polymer solution, from the auxiliary charged plate (Fig. 2a). Thus, the electric fields generated by needle and counter electrode was negligible and the presence of uniform electric field guaranteed.

Although there are various choices of nanofibres material we focused on the deposition of PLGA, a polymer that was studied extensively in our group [12], even though analogous results were obtained by using a star branched three-arm poly( $\varepsilon$-caprolactone). Voltages of the same magnitude but opposite sign were applied to the plates $(+/-16.5 \mathrm{kV})$ while a by far lower induced potential was measured at the needle $(+300 \mathrm{~V})$. The needle possessed the same potential sign of the auxiliary plate. 
http://nanobe.org
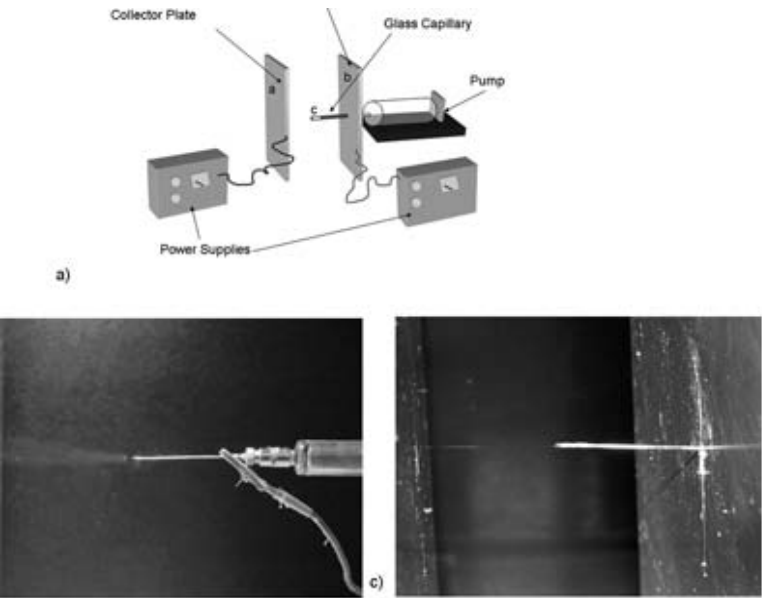

Fig. 2 a) Power supplies are connected to two parallel plates to ensure a homogeneous electric field. The polymer solution and the needle are electrically insulated by a glass capillary; b) Chaotic path of jet by a typical electrospinning set up; c) Straight path of jet obtained by using parallel charged plates and an insulated needle.

The versus of the electric force attracting and stretching the polymer solution into fibers, depended on the distance of the pendant polymer drop from the plates, respectively $d_{a-b}$ and $d_{b-c}$. To avoid the polymer jet to be attracted by the auxiliary plate rather than by the collector plate (a phenomenon that was experimentally observed $), d_{a-c}(4$ $\mathrm{cm}$ ) was kept lower than $\mathrm{d}_{\mathrm{b}-\mathrm{c}}(5 \mathrm{~cm})$ (Fig. 2a).

By using this approach, bending instabilities that typically arises during the process by using a needle plate configuration (Fig. 2b) were avoided. The polymer jet followed a straight trajectory from the tip of the glass capillary to the collector plate (Fig. 2c). The electrospun fiber, continuously depositing in a circumscribed area of about $1 \mathrm{~mm}$, tangled in a three-dimensional structure that extended along the $\mathrm{Z}$ axis for circa $7 \mathrm{~mm}$ before developing instabilities (Fig. 3a).

Even if bending perturbations, commonly considered the main cause of fiber thinning [13], did not seem to take place along the whole path of the jet (Fig. 2c), the diameter of the obtained fiber (Fig. 3b) was in the same range of that obtained by means of a typical electrospinning configuration (Fig. 2b, 4). It seems that
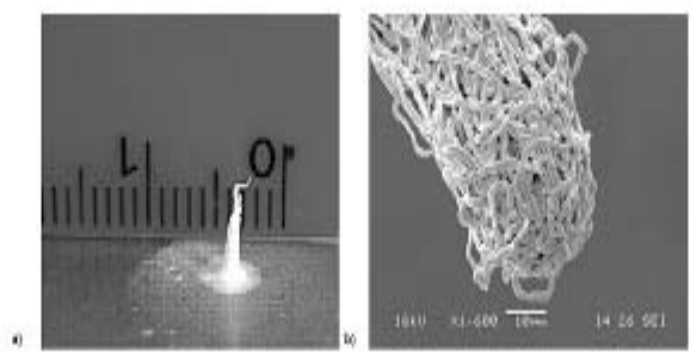

Fig. 3 a) Punctiform deposition of the fiber; b) SEM image of the tip of the punctiform deposition. the simple acceleration of the polymer jet towards the counter electrode along a straight trajectory, together with simultaneous solvent evaporation, was sufficient to achieve a very high reduction in the cross-sectional area of the jet and in the fiber diameter.

By transversally moving the collector plate at a speed of $1 \mathrm{~cm} / \mathrm{sec}$ during fibers deposition, it was also possible to draw a line of tangled fibers (Fig. 5). The motion of the collector avoided both the accumulation of the fibers in the tick conical shape structure shown in Fig. 3a and the consequent development of jet instabilities. This was a first evidence of the feasibility of the method to achieve an accurate control over fiber depositions.

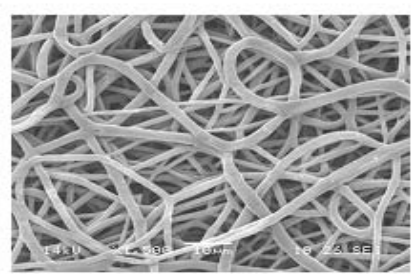

Fig. 4 SEM image of PLGA fibers produced by a typical electrospinning configuration.

Experimental observations of Ref. 14, 15 and numerous related works showed that the electrostatic drawing of low-conductive fluids, such as glycerol or molten polymers, also form the elongated straight jet that is shown in (Fig. 2c). Thus, to confirm the efficacy of the developed system with more conductive solutions, an acetone/DMF (3:2) solution of PLGA was electrospun by using the parallel plates configuration above described. When the solution was directly fed through a metallic

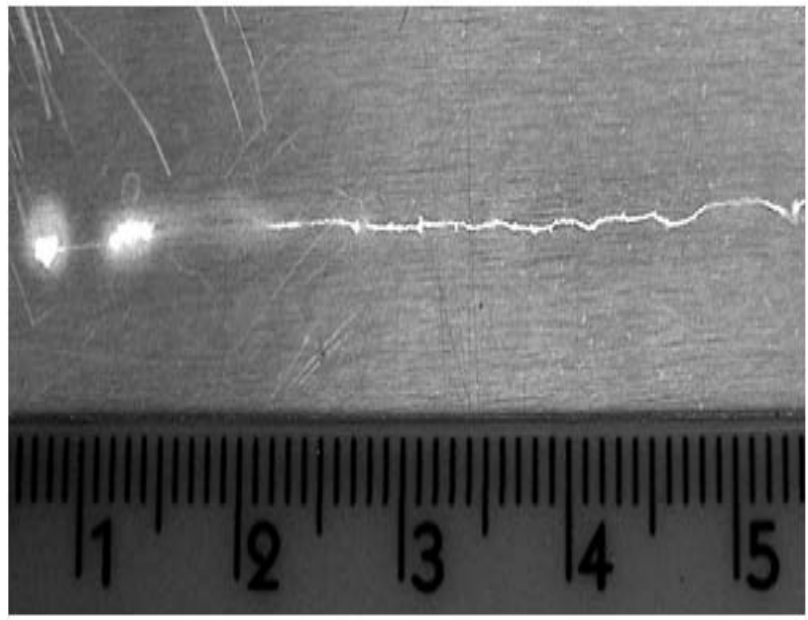

Fig. 5 Picture of a line of tangled fibers as drawn by translating the collector plate. 
needle into the electric field bending instabilities grew rapidly (Fig. 6a, 6b). By insulating the needle with a glass capillary the polymer jet followed a straight trajectory from the droplet to the collector plate (Fig. 6c). As already described for the lower conductive solution of PLGA in acetone, the electrospun fiber deposited in a very circumscribed area and tangled in a three-dimensional structure (Fig. 6d).

\subsection{Controlled deposition by using the concentric cylinders configuration}

A further electrode configuration investigated was that consisting of two concentric cylinders, that alike the parallel plates configuration, forms the geometry of a capacitor. In this case, the inner cylinder consisted of a roto-translating aluminum mandrel concentric
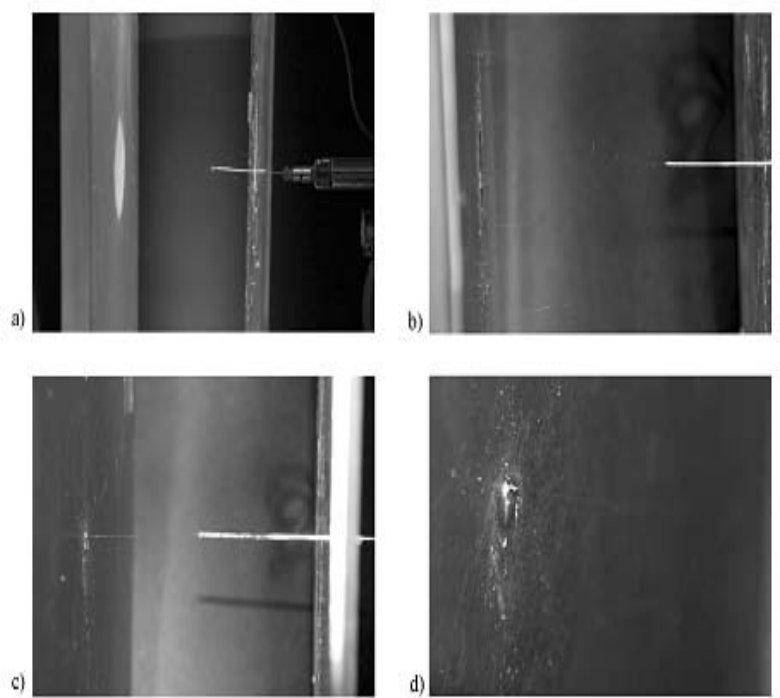

Fig. 6 a) picture of the parallel plate configuration adopted to carry out the experiment; b) electrospinning of PLGA in Acetone:DMF solution by using a metallic needle; c) electrospinning of PLGA in Acetone:DMF solution by insulating the metallic needle with a glass capillary; d) A straight jet of PLGA solution depositing in a very circumscribed area formed a structure that extended along the $\mathrm{Z}$ axis.

to an outer static aluminum hemi-cylinder. The glass capillary, feeding the polymer solution into the electric field, jutted out from the outer hemi-cylinder (Fig. 7). This configuration enabled to carry out the process in a uniform electric field and to collect the fibers on a rapid movable support.

The experimental results obtained by using this configuration corresponded qualitatively to the ones reported above for the parallel plates configuration. The polymer jet electrospun in the uniform electric field deposited on the mandrel without developing any bending instability along its path.

In Fig. 8 is shown the pattern of deposited fibers on the collector. The roto-translational motion of the mandrel

Nano Biomed. Eng. 2011, 3(4), 222-226 http://nanobe.org
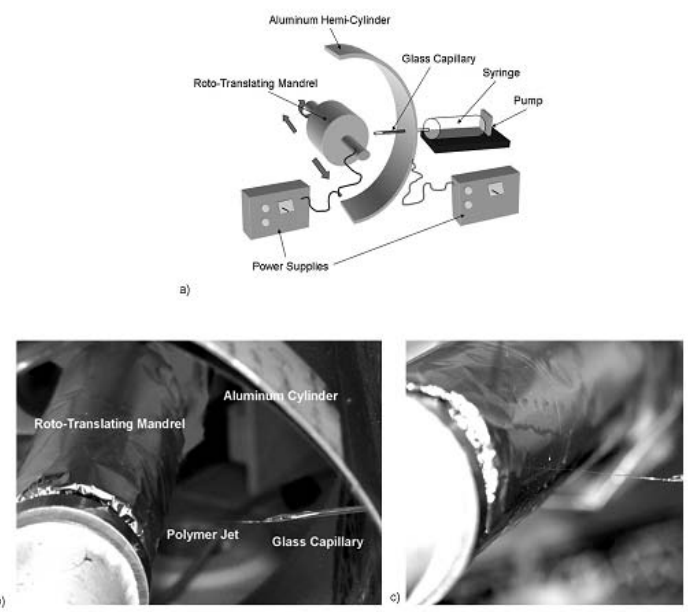

Fig. 7 a) Power supplies are connected to an hemi-cylinder and a concentric rotating mandrel to ensure a homogeneous electric field. Polymer solution and the needle are electrically insulated by a glass capillary; b) Photograph of the apparatus configuration developed to assure operations in a uniform electric field. The glass capillary jutted out from an outer aluminum hemi-cylinder concentric to an aluminum roto-translating mandrel c) The roto-translational motion of the inner cylinder allowed to draw coils consisting of parallel electrospun lines of fibers.

allowed to draw a coil of fibers. The wider coil pitch depended on the translational motion of the mandrel, while the narrower to its traverse reverse.

A straight path of jet incident on a movable substrate gives rise to the deposition of linear nanofibres if the deposition speed and the displacement speed of the support are similar. Fibers were collected at 150, 400, $800 \mathrm{rpm}$ corresponding respectively to linear velocities of 0.3,0.8, $1.6 \mathrm{~m} \mathrm{sec}^{-1}$ (Fig. 9).

Increasing the mandrel rotational speed, the buckling processes were gradually reduced till the achievement of

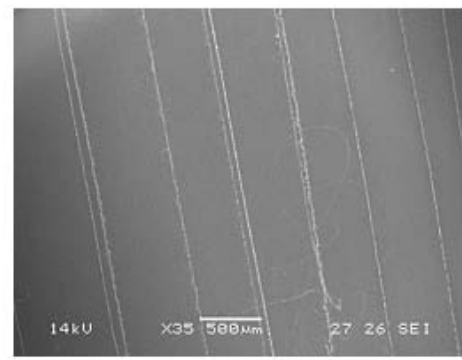

Fig. 8 SEM image of the aligned lines of tangled fibers.

the deposition of a linear fiber at $1.6 \mathrm{~m} \mathrm{~s}^{-1}$, which likely corresponds to the fiber deposition speed. This confirmed that by using the described system it was possible to control the fiber deposition and to obtain straight fibers.

The described configuration allows also to narrow the electrospinning collection area even when an insulating material, that perturbate the electric field, is placed 

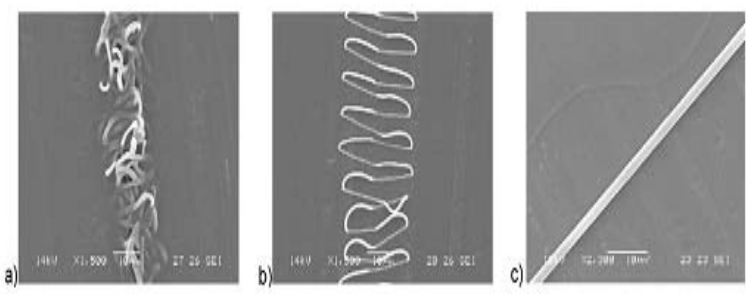

Fig. 9 SEM image of the fiber collected on the rotating mandrel at a) $150 \mathrm{rpm}, \mathrm{b}) 400 \mathrm{rpm}, \mathrm{c}) 800 \mathrm{rpm}$

on the collection area. Mota et al with this processing configuration improved the reliability and reproducibility of the fabrication of dual-scale scaffolds consisting of three-dimensional constructs of aligned poly( $\varepsilon$ caprolactone) (PCL) microfilaments and electrospun poly(lactic-co-glycolic acid) (PLGA) fibers. Beside of improving the fabrication process a better control over the packing density of the collected fibers was achieved [16].

\section{Conclusions}

The electrospinning apparatuses so far proposed for direct-write deposition of fibers, either suppresses the whipping motion by complicated configuration of electrodes or circumvent the first bending perturbations by spinning at very short distances. In the described system, bending perturbations did not develop likely because the polymer solution was fed in a uniform electric field where it was only subjected to a constant and regular attraction force towards the counter electrode. Furthermore, the low charge of the polymer solution could not give rise to bending perturbations that imposes a spiroidal movement to the jet. Despite the lack of bending instabilities, a very high reduction in the cross-sectional area of the jet and in the resulting fiber diameter was obtained. Conventional electrospinning using flat collector plates as a counter electrode leads to the formation of flat meshes. For the first time our system gave the possibility to produce meshes that extended in height more than in width. The results shown in this work are expected to give a contribution for a better theoretical understanding of the process and to offer new opportunities to extend the applicability of electrospinning.

\section{Acknowledgment}

The work in the present manuscript was performed within the framework of the FP6 EC-fundedNoE project "EXPERTISSUES" NMP3-CT-2004-500283 and the Italian FIRB Internazionale 2004 project prot. RBIN043BCP. Thanks are due to Dr. Dinuccio Dinucci who records pictures during electrospinning.

\section{References}

1. Fridrikh SV, Yu JH, Brenner MP, Rutledge GC. Controlling the fiber Diameter during Electrospinning. Phys. Rev. Lett. 2003;90:1-4 doi: http://link.aps.org/doi/10.1103/PhysRevLett.90.144502

2. Carnell LS, Siochi EJ, Wincheski RA, Holloway NM, Clark RL. Electric Field Effects on Fiber alignment Using an Auxilary Elctrode During Electrospinning.Scr. Mater. 2009; 60: 359- 361. doi: http://dx.doi.org/10.1016/j.scriptamat.2008.09.035

3. Li D, Ouyang G, McCann JT, Xia Y. Collecting Electrospun Nanofibers with Patterned Electrodes Nano Lett. 2005;5: 5. 10. doi: 1021/n10504235

4. Sangamesh GK, Syam PN, Roshan J, MaCalus VH, Cato TL. Recent Patents on Electrospun Biomedical Nanostructures: An Overview. Recent Patents on Biomedical E. 2008; 1: 68-78. doi: 10.1007/9781-61779-052-2 16

5. Reneker DH, Yarin AL. Electrospinning Jets and PolymerNanofibers. Polymer 2008; 48: 2387-2425. doi:10.1016/S1359- 029 4Ž03.000049

6. Sun D, Chang C, Li S, Lin L.Near Field Electrospinning. NanoLett. 2006; 6: 839-842. doi:10.1063/1.2975834

7. Hellmann C, Belardi J, Dersch R, Greiner A, Wendorff, JH, Bahnmueller S. High Precision Deposition Electrospinning of nanofibers and nanofiber nonwovens Polymer 2009; 50: 1197- 1205.

8. Teo WE, Ramakrishna S. A review on Electrospinning Design and Nanofibre Assemblies. Nanotechnology 2006; 17: 89-106. doi:10.1088/0957-4484/17/14/R01

9. Shin YM, Hohman MM, Brenner MP, Rutledge GC. Experimental Characterization of Electrospinning: the Electrically Forced Jet and Instabilities. Polymer 2001; 42, 9955-9967. doi:10.1088/0957-448 4/17/14/R01

10.Buyan NN, Chen JC, Chen I, Farboodmanesh S. Electrostatic Effects on Electrospun Fiber Deposition and Alignment in Poly meric Nanofibers (ACS Synposium Series 918) DH Reneker H Fong 2006

11.Errico C, Piras AM, Detta N, Puppi D, Chiellini F, Chiellini E. Un nuovo metodo e apparato dielettrofilatura per la produzione di nano-microfibre polimeriche unidirezionate RM2009A00286

12Puppi D, Piras AM, Detta N, Dinucci D, Chiellini F. Poly(lactic- coglycolic acid) electrospun fibrous meshes for the controlled release of retinoic acid. Acta Biomaterialia. 2010;1258-1268. doi:10.1016/j.a ctbio.2009.08.015

13.Reneker DH, Yarin AL, Koombhongse Bending instabilities of electrically charged liquid jets of polymer solutions in electrospinning Journal of applied Physics 2000; 87(9): 4531-4547. doi:10.1063/1.373532

14.Rangkupan R and Reneker DH Electrospinning process of molten polypropylene in vacuum J. Met. Mater. Miner. 2003;12: 8187.

15. Zhou H, Green, TB, Joo YL. The Thermal Effects onElectrospinning of Poly-lactic Acid Melts Polymer. 2006; 47: 7497-7505.

16. Mota C, Puppi D, Dinucci D, Errico C, Bártolo P, Chiellini FDualScale Polymeric Constructs as Scaffolds for Tissue Engineering Materials. 2011; 4: 527-542. doi:10.3390/ma4030527

Copyright:(c) 2011 Cesare Errico et al. This is an open-access article distributed under the terms of the Creative Commons Attribution License, which permits unrestricted use, distribution, andreproduction in any medium, provided the original author andsource are credited. 